\title{
A vigilance avoidance account of spatial selectivity in dual-stream emotion induced blindness
}

\author{
Matthew Proud $^{1,2} \cdot$ Stephanie C. Goodhew ${ }^{1} \cdot$ Mark Edwards $^{1}$
}

Published online: 2 January 2020

(C) The Psychonomic Society, Inc. 2020

\begin{abstract}
Emotion-induced blindness (EIB) is the impaired processing of neutral images when they are preceded in close temporal proximity by an emotive distractor. Dual-stream EIB contains two visual streams so the distractor and target can appear in either the same or opposite streams. Results from these studies suggest that the EIB effect is spatially localised. That is, for EIB to occur, the target must appear in the same stream as the distractor. An early spatially localised attention model has been proposed to account for these results. However, such an explanation is incompatible with the involvement of a high-level attentionalbottleneck in the processing of emotive stimuli. Here we propose and test an alternative account of the dual-stream EIB findings - specifically, a vigilance-avoidance (VA) account that is compatible with the high-level attentional bottleneck. We tested this model by using both negative and positive distractors and by measuring the trait anxiety of the participants. VA predicts that spatial localisation of the EIB effect would only occur with negative (threat-based) distractors with participants who have high levels of trait anxiety and that for all other conditions EIB would be obtained in both streams, while the early-localised-attention account predicts spatial localisation for both types of distractors, regardless of trait-anxiety levels. Results supported the VA model. This means that the results of EIB studies as a whole are consistent with conventional-attentional-bottleneck theories and therefore support the use of the EIB paradigm to investigate the impact of emotive stimuli on attentional processing.
\end{abstract}

Keywords Attention $\cdot$ Emotion $\cdot$ Emotion induced blindness $\cdot$ Anxiety $\cdot$ Vigilance avoidance

\section{Introduction}

For most of us, vision provides the dominant source of information about the outside world. However, we cannot simultaneously process all of the information in typical visual scenes. Visual attention prioritises information for selective enhanced processing (Carrasco, 2011). In this process, emotive stimuli, especially those associated with threat and reward, are automatically given high attentional-priority, and so get selected by visual attention even when they are not relevant to our current tasks (Most, Smith, Cooter, Levy, \& Zald, 2007; Ohman, Flykt, \& Esteves, 2001).

Mark Edwards

mark.edwards@anu.edu.au

1 Research School of Psychology, Australian National University, Canberra, ACT, Australia

2 Current address School of Psychology, University of Queensland, St Lucia, QLD, Australia
A useful experimental paradigm for investigating this automatic prioritisation of emotive information is emotion induced blindness (EIB). Developed by Most and colleagues (Most, Chun, Widders, \& Zald, 2005), EIB consists of the rapid-serial-visual presentation (RSVP) of images in which a target is embedded amongst filler images with the task being to indicate the orientation of the target. A distractor is presented at varying temporal positions before the target, and it can be either an emotionally evocative (hereafter 'emotive') or emotionally neutral image. The emotive image can either be negative (e.g., images depicting physical threat) or positive (e.g., erotica). Performance is impaired when the target closely follows the distractor, but most importantly, the impairment is greatest following emotive distractors. This differential impairment in performance between emotive and neutral distractors is called the EIB effect, and is typically greatest when the target is the second image after the distractor (i.e. $\operatorname{lag} 2$ ).

The results of these EIB studies are consistent with conventional attentional-bottleneck theories of attention. These state that following an initial capacity-free encoding of 
information, visual attention selects objects for capacitylimited processing and the allocation of these resources is not spatially localised (Carrasco, 2011). Once stimuli enter this capacity-limited stage, they remain there until their processing is complete. This process takes around $500 \mathrm{~ms}$ (Chun $\&$ Potter, 1995) after which these attentional resources can be allocated to another stimulus. Thus, in an EIB stream, attention automatically processes the emotive stimuli, so when a target is subsequently presented within this temporal window, attention cannot be allocated to it, and it gets overwritten by the subsequent images in the RSVP stream and its representation is lost (Kennedy \& Most, 2015).

While the results of single-stream EIB studies are consistent with high-level bottleneck theories of attention, the results of dual-stream EIB studies present a challenge to them. Dualstream EIB stimuli contain two simultaneous and spatially offset RSVP streams, meaning the distractor and target can be presented in either the same or opposite streams. EIB is only observed when they are in the same stream (Kennedy, Pearson, Sutton, Beesley, \& Most, 2018; Most \& Wang, 2011; Wang, Kennedy, \& Most, 2012; Wang \& Most, 2016). By itself, the cognitive-level (i.e. non-spatially localised) bottleneck cannot account for these results because if emotive stimuli tapped this bottleneck, then there would also be impaired performance in the opposite stream. Instead, these results have been explained in terms of an early attentional mechanism. Specifically, an early-neural competition model of EIB in which stimuli that occur closely in both space AND time compete to drive the same neural response of a shared retinotopic region (Most \& Wang, 2011; Wang et al., 2012). We call this the early-localised-attention account, and it is compatible with other early-neural competition models (Desimone \& Duncan, 1995; Keysers \& Perrett, 2002; Wyble \& Swan, 2015).

It is important to note, however, that while this spatially localised-attention account can explain the dual-stream data, it is inconsistent with the involvement of the conventional attentional-bottleneck in dual-stream EIB. This is because if the attentional bottleneck was operating during dual-stream EIB, this bottleneck would continue to prioritise emotive stimuli over other stimuli that occur close in time to them (regardless of whether they were in the same stream or not), and so opposite-stream sparing should not occur. For sparing to occur, it implies that late-stage emotive prioritisation by the attentional bottleneck cannot occur in dual-stream EIB, and hence, presumably, in EIB as a whole. While this may be the case, it seems unlikely that the attentional bottleneck would not apply for emotive stimuli. Therefore, is it possible to account for the dual-stream EIB data using the high-level attentional-bottleneck? In considering this possibility, it is worth reconsidering the interpretation of the data and the nature of the stimuli used in the dual-stream EIB studies.
The dual-stream EIB data have been interpreted as indicating EIB impairment in the same-stream condition and no impairment in the opposite-stream condition. However, could it actually reflect a selective performance improvement in the opposite-stream condition? If so, this improvement for targets presented in the opposite stream at lag 2 (i.e. $200 \mathrm{~ms}$ after the distractor) would run counter to the idea that emotive stimuli always capture and hold attention. In relation to this, note that this spatially localised pattern of impairment has only been obtained with threat-based distractors. In contrast, the single-stream EIB effect is also obtained with positive distractors (Ciesielski, Armstrong, Zald, \& Olatunji, 2010; Most et al., 2007). Therefore, do the dual-stream EIB results reflect a threat-specific process, rather than reflecting the processing of emotive stimuli in general? One possible mechanism is vigilance avoidance.

Vigilance avoidance (VA) is a cognitive reaction to threat by individuals with high trait-anxiety levels, which manifests as enhanced initial visual engagement with threat-based stimuli followed by rapid spatial disengagement from that threat (Koster, Crombez, Verschuere, Van Damme, \& Wiersema, 2006). The theory was developed to explain an apparent repressive coping style amongst high-trait-anxious people. Following the rapid automatic orientation of attention to threat-based stimuli (vigilance) they then rapidly redirect attention away from that stimulus (avoidance) in an attempt to reduce associated anxiety (Mogg, Bradley, Miles, \& Dixon, 2004). Trait anxiety is a key feature of VA, and is defined as a person's predisposition to respond in an anxious manner over time (Endler \& Kocovski, 2001).

Can VA explain the dual-stream-EIB data? When presented with a threatening distractor, a high-trait-anxious participant would initially engage with it, but then rapidly redirect their attention away from the threat. Given the presence of the two RSVP streams, it is likely that they would redirect their attention to the second stream. This rapid disengagement with the emotive stimulus and redirection of attention from the same to the opposite stream would account for both impaired target performance for that same stream, and the better performance in the opposite-stream conditions. Accounting for the dualstream EIB data via VA would mean that automaticprioritisation of emotional stimuli combined with the highlevel attentional-bottleneck would account for the results of single- and dual-stream EIB studies, negating the need for the early spatiotemporal account mechanism.

Both the early-localised-attention and VA accounts are compatible with the current dual-stream EIB data. Where they differ is in their predictions regarding the effects of positivevalenced distractors and trait-anxiety level with negativevalenced distractors. The early-localised account predicts spatially localised EIB regardless of the valence of the emotional distractors and the participant's trait-anxiety level. In contrast, according to the VA account, positive distractors would not 
elicit avoidance (since they are not threatening) and negative distractors would only elicit avoidance in the highly anxious group. Thus, VA predicts spatially localised EIB only for negative distractors for the highly anxious group and for all other conditions (negative distractors, low-anxiety participants and positive distractors, all participants) it predicts EIB in both streams. Therefore, the current study pitted the two theories against one another by using both positive and negative distractors in a dual-stream EIB experiment and by also measuring the trait anxiety of participants.

\section{Method}

\section{Participants}

One hundred and six students from the Australian National University (ANU) participated in this study; 93 were female, and the average age of the participants was 21.9 years $(\mathrm{SD}=$ 4.3). Participants were recruited via ANU's online Psychology Research Participation Scheme (SONA) and compensated for their time with either course credit or $\$ 10$ cash payment. All participants gave voluntary, informed consent, and the study was approved by the ANU Human Research Ethics Committee.

\section{Apparatus and stimuli}

Dual-stream EIB All stimuli were presented on a cathode-raytube monitor, with a resolution of $1,280 \times 1,024$ pixels and a refresh rate of $100 \mathrm{~Hz}$ via MATLAB's Psychophysics Toolbox (Mathworks, Natick, MA, USA). Participants were seated and a chinrest was used to maintain a viewing distance of $550 \mathrm{~mm}$. Images, stimulus parameters and procedures were based on previous EIB studies by Most and colleagues (Kennedy \& Most, 2015; Most \& Wang, 2011; Wang \& Most, 2016). Stimuli were presented on a mid-grey background and consisted of coloured photographs measuring $11.2^{\circ}$ (height) by $18.4^{\circ}$ (width). Target images consisted of 128 landscape or architectural photographs sourced freely from the internet that were rotated $90^{\circ}$ to both the left and the right, forming 128 image pairs a total pool of 256 images. Each target was only presented once to each participant.

Filler images consisted of 374 landscape or architectural images, which were also sourced from the internet. These images were conceptually identical to the target images in their content, but were always vertically orientated. In order to cater for the required number of filler images $(5,808$ in total) the 374 filler images were resampled after every 16 trials.

The neutral, negative and positive distractor images depicted either people or animals in order to ensure they were conceptually distinct from the filler and target image sets (which contained no people), and were primarily sourced from the International Affective Picture System (IAPS) (Lang, Bradley, \& Cuthbert, 2005) and supplemented where necessary by images from the Open Affective Standardized Image Set (OASIS) (Kurdi, Lozano, \& Banaji, 2017). Given that the OASIS and IAPS database images are rated on different scales (7- vs. 9-point scale), all image ratings were standardised by converting their raw scores into a percentage of their maximum possible score prior to image selection.

There were 64 negative images, including threatening animals, scenes of assault/threat and gore. Positive images consisted of 64 erotic scenes, depicting either heterosexual couples engaged in intimate acts or single-person nudes. The positive and negative image sets were matched in terms of arousal (mean arousal for negative and positive was comparable at $73.62 \%(S D=4.40 \%)$ and $70.47 \%(S D=5.10 \%)$ respectively), and their valence was suitably polarised at $27.75 \%(S D=9.68 \%)$ for negative and $72.49 \%(S D=$ $5.26 \%$ ) for positive. The neutral images (128) contained people and animals, and were selected based on their low mean arousal, and moderate (neither high nor low) valence ratings (arousal $M=42.54 \%, S D=6.06 \%$, and valence $M=58.46 \%$, $S D=7.59 \%)$.

It is important to consider the reliability of experimental measures being used in individual-differences research (for a review and discussion, see Goodhew \& Edwards, 2019). Raw scores at lag 2 in EIB have been found to have intra-class correlation coefficients between .8 and .9 for the negative and neutral distractor conditions (Onie \& Most, 2017). Therefore, EIB has excellent reliability.

State-Trait Anxiety Inventory (STAI) Both state- and traitanxiety levels were measured with Spielberger's STAI (Spielberger, Gorsuch, Lushene, Vagg, \& Jacobs, 1983). The STAI is a widely utilised self-report measure of anxiety, consisting of two forms, a Y-1 state-anxiety and a Y-2 traitanxiety. Each form consists of 20 questions that are rated on a 4-point scale ( $1=$ almost never, to $4=$ almost always) with possible scores ranging from 20 to 80 on each form. Higher scores on this measure indicate higher levels of anxiety. A reliability meta-analysis of 75 studies utilising the STAI showed high internal consistency, with a mean Cronbach's Alpha for state-anxiety of .91 $(S D=.05)$ and trait-anxiety of $.89(S D=.05)$ (Barnes, Harp, \& Jung, 2002). They also reported a satisfactory mean test-retest coefficient for traitanxiety of $.88(S D=.05)$, but a lower and more variable coefficient of $.70(S D=.20)$ for state-anxiety, which is expected due to the transitory nature of state-anxiety as a construct.

Demographic and Screening Questionnaire Basic demographic information including age and sex was recorded. Information regarding the participant's history and current experience with neurological impairments, substance use and visual impairment was also recorded and their data excluded 
from the final analysis if they responded positively to any of these questions due to the possibility that they may have adversely affected task performance.

\section{Procedure and design}

The experiment was a 2 (Block type, Positive and Negative) $\times$ 2 (target Stream location, Same and Opposite to the distractor) $\times 2$ (Distractor type, Neutral and Emotive) design, with trait anxiety as the covariate.

The experiment commenced with the dual-stream EIB task. Participants completed an initial practice session (five trials) in which no emotive distractor was used and image presentation was slowed by $250 \%$. If the participant could not successfully identify all five targets the practice session was repeated up to five times. If they failed to correctly identify all five targets after five attempts, they were allowed to continue the study but their data were excluded from the final analysis.

The main experiment consisted of four blocks. Two blocks contained positive (erotic) and neutral distractors (Positiveblock condition) and the other two contained negative (threat) and neutral distractors (Negative-block condition). Block order was counterbalanced across participants. Each block consisted of 64 trials with a randomised order but equal number of emotive and neutral distractor trials in each -32 emotive and 32 neutral. This resulted in 256 total trials for the whole experiment (64 positive, 64 negative and 128 neutral). Each block had a participant-controlled rest break after every 16 trials.

Each experimental trial commenced with a central, black fixation-dot presented for $500 \mathrm{~ms}$, followed by a blank screen for $200 \mathrm{~ms}$, and then two simultaneous streams of images positioned centrally above and below the position of the original fixation dot and separated from each other by $2^{\circ}$. Each stream consisted of 12 images, so 24 images in total, including 22 fillers, one distractor and one target. Each image was displayed for $100 \mathrm{~ms}$ and was immediately replaced by the next image. The distractor appeared equally often at positions $2,4,6$ or 8, and appeared equally often in either the upper or lower stream. The target appeared equally often in the same and opposite stream to the distractor and always appeared two positions after the distractor, given that lag 2 has been identified as the reliable position for the EIB effect (Most et al., 2005). At the end of each trial the participant indicated the orientation of the target image via a keyboard response.

At the conclusion of the dual-stream EIB task, participants were shown a slideshow of ten positive and happy full-screen images - such as kittens and children playing - for $3 \mathrm{~s}$ each, to mitigate any lingering affective impact of the experimental images. Finally, participants completed a paper-based version of the STAI, commencing with Form Y-1 followed by Y-2 and then the Demographic and Screening Questionnaire.

\section{Results and discussion}

\section{Data screening}

Of the 106 participants, 20 were excluded from final analysis due to either indicating a positive response to exclusionary questions included in the Demographic and Screening Questionnaire and/or failing to meet performance cut-offs for the dual-stream EIB task. ${ }^{1}$ The final sample included 86 participants ( 74 females) with an average age of 21.75 years $(S D=4.04)$. This sample size was in excess of the 85 required to detect a medium effect of $f=.15$, at $1-\beta=.8$ and $\alpha=.05$, according to an a priori power analysis conducted via GPower 3. There were no out-of-range values, no missing values and no univariate outliers detected via $z$-scores $( \pm 3.29, p<.001)$. Raw data are publicly available here: https://osf.io/dhuqw/.

\section{Analysis of covariance (ANCOVA)}

Prior to analysis both covariates, state- and trait-anxiety, were centred by subtracting the mean from each score to make them suitable for ANCOVA. A 2 (Block) $\times 2$ (Target Stream location) $\times 2$ (Distractor Type), repeated-measures ANCOVA, with trait-anxiety as the covariate, was conducted to determine if trait-anxiety had a different impact on the spatial localisation of the EIB effect between the two blocks. This revealed a significant Block $\times$ Stream $\times$ Distractor $\times$ Anxiety interaction effect, $F(1,84)=5.03, p=.028, \eta_{p}{ }^{2}=.06,95 \%$ CI $[.00, .17]$. In order to investigate the nature of this effect, subsequent planned ANCOVAs, inclusive of a Bonferroni correction to $\alpha=.025$, were conducted for each Block condition.

\section{Negative block}

Figure 1 illustrates that target identification was impaired following negative distractors (i.e., a standard EIB effect) in both the same and opposite streams. This pattern was confirmed by a 2 Stream (Same and Opposite) $\times 2$ Distractor Type (Neutral and Threat) repeated-measures ANCOVA, with trait-anxiety as the covariate. This revealed a significant main effect of Distractor Type, but a non-significant Stream $\times$ Distractor interaction $(F<1)$. Furthermore, the critical Stream $\times$ Distractor $\times$ Anxiety interaction effect was significant, $F(1,84)=9.93, p$

\footnotetext{
${ }^{1}$ Two participants were excluded as a result of indicating that they had consumed a substance in the past $24 \mathrm{~h}$ that likely impacted their cognitive performance, one participant was excluded for failing to adequately complete the dual-stream EIB task due to illness and another 17 were excluded for failing to reach the mean performance cut-off of $60 \%$ across neutral trials. This performance cut-off was enforced in order to ensure that participants were able to perform suitably above chance level $(50 \%)$ in response to the un-emotive neutral distractors so that they demonstrated the potential to experience an EIB effect without risking the effect being consumed by chance (Most \& Wang, 2011).
} 

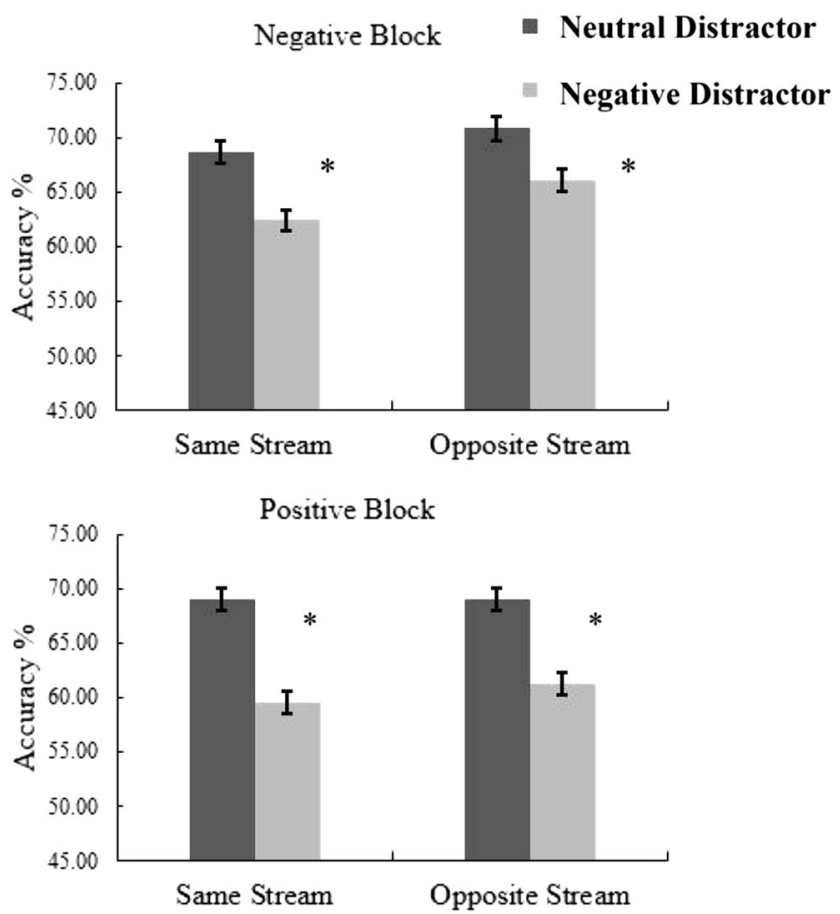

Fig. 1 Upper: Target identification accuracy for the Same- and DifferentStream conditions for all participants in the Negative-distractor condition. Lower: Target identification accuracy for the Same- and DifferentStream conditions for all participants in the Positive-distractor condition. An emotion-induced blindness (EIB) effect was obtained for both distractors in both streams

$=.002, \eta_{p}{ }^{2}=.11,97.5 \% \mathrm{CI}[.01, .26]$. This indicates that the spatial localisation of the apparent EIB effect for Negative distractors changed as a function of participants' anxiety.

To demonstrate this effect of anxiety, a spatial-localisation index was created. This index quantified the extent to which the magnitude of the EIB effect (i.e. the difference in performance between the Neutral and Negative distractor conditions) differed between the Opposite- and Same-Stream conditions. The index was equal to the (EIB magnitude for the Same-Stream condition) minus (EIB magnitude for the Opposite-Stream condition). This means that positive values on this index indicate greater EIB magnitude in the same stream (or less EIB in the opposite stream), and hence a greater spatial-localisation effect. This spatial-localisation index was then correlated with trait-anxiety (see Fig. 2), revealing a significant Pearson correlation of $r(84)=.33, p=.002,95 \%$ CI $[.13, .51] .^{2}$

In order to test the predictions of the two accounts with respect to the effect on EIB of anxiety for negative distractors,

\footnotetext{
$\overline{{ }^{2} \text { Given that VA }}$ only proposes a role for trait-anxiety, an analysis was conducted with the centred and transformed state-anxiety variable as the covariate. A full 2 Block (Negative and Positive) $\times 2$ Stream (Same and Opposite) $\times 2$ Distractor Type (Neutral and Emotive) ANCOVA with the state-anxiety as the covariate revealed a non-significant Block $\times$ Stream $\times$ Distractor $\times$ Anxiety interaction effect $(F<1)$, indicating that spatial localisation of the EIB effect did not depend upon levels of state-anxiety. This result further supports the VA account of EIB.
}
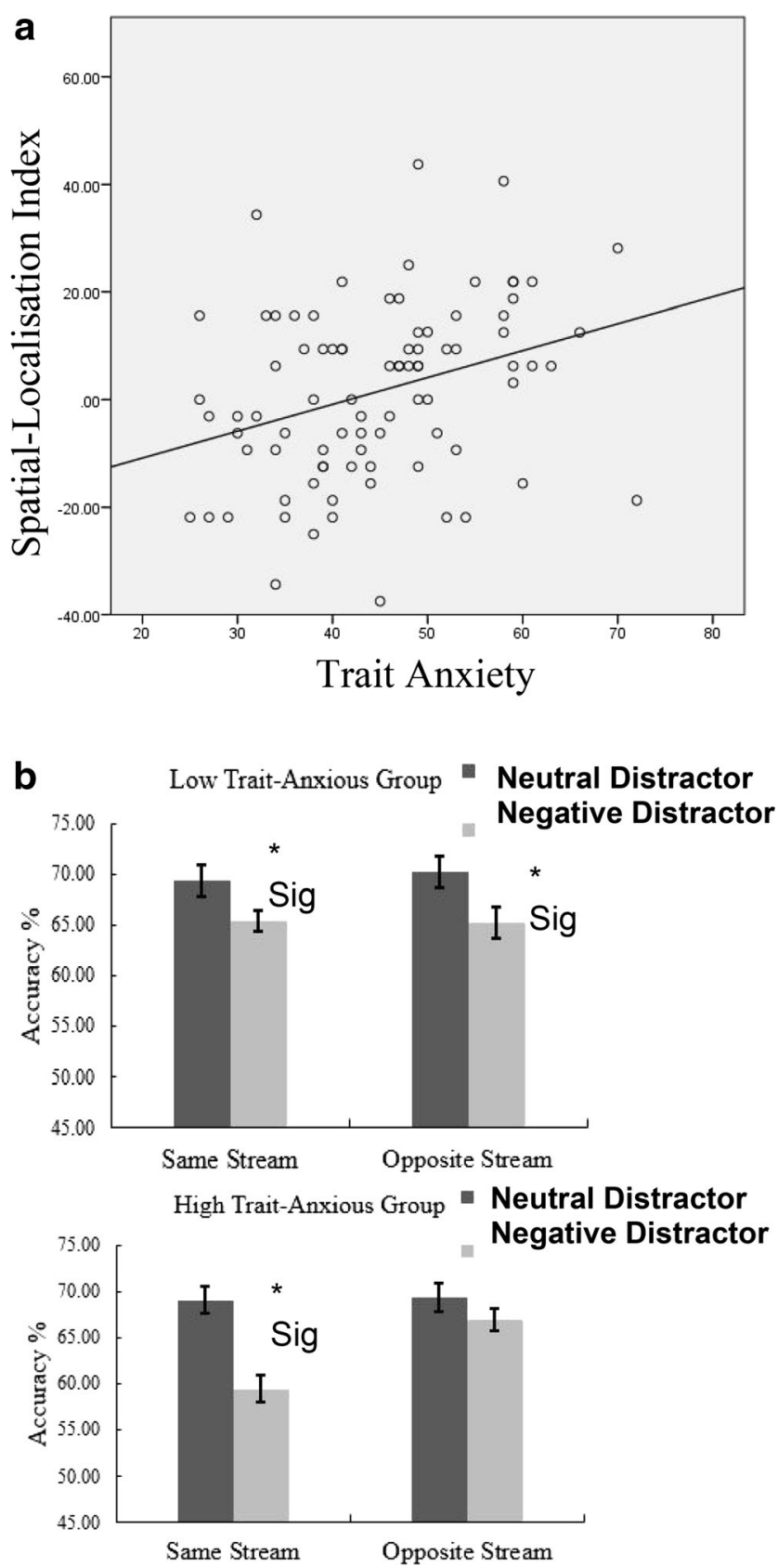

Figure 2 (a) Index of the Spatial-Localisation index plotted against traitanxiety for the negative distractor. Positive values of the index indicate a great emotion-induced blindness (EIB) effect for the Same-Stream condition compared to the Different-Stream condition for the negative distractors. The significant correlation of 0.33 indicates that spatiallocalisation of the EIB effect for negative distractors does depend upon the trait-anxiety level of the participant. (b) Upper: Target identification accuracy with the negative distractor for the Same- and Different-Stream conditions for participants who had low trait-anxiety. Significant EIB was obtained in both streams. Lower: Target identification accuracy with the negative distractor for participants who had high trait-anxiety. Significant EIB was obtained only in the same stream

a median split of the sample based on trait-anxiety was conducted. A 2 Stream (Same and Opposite) $\times 2$ Distractor Type (Neutral and Threat) repeated-measures ANOVA was run on 
each group. For the low-anxiety group it revealed a nonsignificant Stream $\times$ Distractor interaction effect, $F(1,42)=$ $3.25, p=.079, \eta_{p}{ }^{2}=.07,95 \% \mathrm{CI}[.00, .25]$, indicating that EIB was of the same magnitude across stream conditions. For the high-anxiety group it revealed a significant Stream $\times$ Distractor interaction effect, $F(1,42)=8.42, p=.006, \eta_{p}{ }^{2}=$ $.17,95 \%$ CI $[.02, .36]$, indicating that EIB was of a different magnitude across stream conditions for the high-anxious group. To investigate the nature of this effect, two pairedsample $t$-tests with Bonferroni correction were conducted, revealing significant EIB in the Same-stream condition, $t(43)=$ $4.83, p<.001, d=0.73,97.5 \%$ CI $[.34,1.12]$, but not the Opposite-stream condition, $t(43)=1.52, p=.136, d=0.23$, $97.5 \%$ CI $[-.11, .57]$.

This pattern of EIB in both streams for the low-anxiety group and EIB in same stream only for the high-anxiety group is consistent with the VA account and inconsistent with the spatially-localised-attention account.

\section{Positive block}

As can be seen in Fig. 1, target accuracy was reduced following the erotic distractor compared with a neutral distractor, and this was true for both streams (i.e. not spatially localised). This was confirmed by a 2 Stream (Same and Opposite) $\times 2$ Distractor Type (Neutral and Erotica) repeated-measures ANCOVA, with trait-anxiety as the covariate. This revealed a non-significant Stream $\times$ Distractor $\times$ Anxiety interaction effect $(F<1)$, indicating that anxiety had no impact on the amount of spatial localisation of the EIB effect for Positive distractors. A significant main effect of Distractor Type, $F(1$, $84)=102.51, p<.001, \eta_{p}{ }^{2}=.55,97.5 \%$ CI $[.38, .66]$, revealed that performance was significantly worse following erotic distractors versus neutral distractors, indicative of overall positive-distractor-induced EIB. The Stream $\times$ Distractor interaction effect was non-significant $(F<1)$, indicating that significant EIB occurred equally in both stream conditions. Taken together, these results support the VA theory, which predicts that positive-distractor-induced EIB will occur in both stream conditions and that trait-anxiety will have no impact on this effect. They are inconsistent with the earlylocalised-attention account in that it would also predict spatial-localisation for positive distractors, regardless of trait anxiety.

\section{General discussion}

It has previously been shown that the impaired performance in dual-stream EIB studies is both temporally and spatially localised. This led to the proposal of an early-neural-competition model, asserting that neurons early in the visual system prioritise their response towards emotive over non-emotive stimuli whenever the stimuli appear in close temporal and spatial proximity to each other (Most \& Wang, 2011). However, such an account is inconsistent with the involvement of the high-level attentional bottleneck in the processing of emotive stimuli, since if it was involved, EIB should regardless of which stream the target is in relative to the distractor. Given that dual-stream EIB has only been previously tested with threat-based distractors, another possibility is that the spatiality localised impairment is due to a threatspecific mechanism, namely, vigilance avoidance. Here we pitted these two theoretical accounts against one another, using both negative and positive distractors and measuring the trait anxiety of the participants. The spatially localised account predicts that spatially localised EIB would occur for both types of distractors and for all participants. The VA account, on the other hand, predicts that spatially localised EIB should only occur with negative distractors for the highanxiety group, while all other conditions (negative distractors low-anxiety group and positive distractors all participants) should result in EIB in both streams. The pattern of results obtained in the present study is consistent with these predictions of the VA account and inconsistent with those of the spatially localised account (Wang et al., 2012).

Note, a corollary of the prediction of the VA account that EIB would only occur in the opposite stream for the lowanxiety group is that the high-anxiety group should have a performance advantage with the negative distractors in the opposite stream. That is, EIB magnitude in the opposite stream with the negative distractors should be lower for the high-anxiety group compared to the low-anxiety group. We investigated this by determining whether there was a significant interaction between valence (negative vs. neutral) and anxiety in the opposite stream. The interaction was not significant, $F(1,84)=2.68, p=.106, \eta_{\mathrm{p}}{ }^{2}=.031$, (whereas it was for the same-stream condition, $F(1,84)=6.01, p=.016, \eta_{\mathrm{p}}{ }^{2}=$ .067). This means that while there was a significant EIB in the opposite stream for the low-anxiety group and not for the high-anxiety group, when we directly compared their relative magnitude, that difference was not statistically reliable. A likely reason for this is that the magnitude of EIB in opposite stream for the low-anxiety group was already reduced. Previous single-stream EIB studies have suggested that EIB magnitude may vary as a function of anxiety or anxietyrelated factors (Most et al., 2005; Onie \& Most, 2017). Therefore, the reduced EIB magnitude in the opposite stream for the high-anxiety group was being compared to an already EIB magnitude for the low-anxiety group. The idea of increased EIB magnitude for negative distractors as a function of anxiety is also supported by our study in that we found greater EIB magnitude for the positive distractors compared to the negative distractors, as supported by a significant interaction between Block and Distractor from the main ANCOVA $(p=.010)$. This was such that the mean effect of the Distractor 
valence (emotive versus neutral) was greater for the Positive block $(M=8.8 \%)$ than for the Negative block $(M=5.5 \%)$.

It is interesting to note that, unlike the previous dual-stream studies, we did not find a spatially localised effect with the negative stimuli at the group level (Kennedy et al., 2018; Most \& Wang, 2011; Wang \& Most, 2016). This does not impact the conclusions of the current study, given our finding that spatial localisation with negative stimuli is dependent upon trait anxiety, such that only high-anxious individuals demonstrate the effect, and does not occur for positive stimuli. However, it does suggest that the overall magnitude of the spatial-localisation effect was smaller in our study. There are several possible reasons why this may have occurred. One possibility is that the participants in our study had lower overall trait-anxiety than those who participated in the previous studies. The previous studies did not report trait-anxiety levels so we cannot check this. However, given that the mean traitanxiety score for our sample was 0.5 standard deviations above the (already elevated) college/university norms (Spielberger et al., 1983), this seems unlikely. Another possibility is that our negative stimuli were not as threatening as those used in the previous studies. We used three subclasses of threat type (threatening animals, scenes of assault/threat and gore) while previous studies used a single threat type. It is possible that mixing different threats minimises the effect, or that some types of threatening stimuli are stronger than others. Finally, it is possible that mixing the positive and negative stimuli in each experimental sequence minimised the effect. Previous studies did not use positive stimuli, and therefore had exclusively negative-valence blocks. It will be interesting for future research to examine these possibilities. However, most importantly, the present study did find evidence of both EIB and, more specially, a spatially localised effect, and identified a factor that modulates this effect, which is entirely consistent with the vigilance avoidance account.

The results of the present study mean that dual-stream EIB can be explained within a high-level attentional-bottleneck framework, without the need for the involvement of an early, spatially localised attentional mechanism. This means that EIB like the attentional blink, reflects the functioning of a central attentional-bottleneck. Where they differ, however, is whether the stimulus that produces the subsequent decrement in performance is task relevant or not. This suggests that EIB uniquely captures the automatic prioritisation of emotive content. Moreover, recent work has shown that EIB explains unique variance in negative affect (Onie \& Most, 2017), thereby highlighting the utility of EIB in our understanding of the emotion-cognition nexus.

In conclusion, vigilance avoidance can explain the apparent spatial localisation of dual-stream EIB. This was supported in the present study, which revealed no spatial localisation of EIB following positive distractors, and that for negative distractors, spatial localisation depends upon participants having high levels of trait anxiety. This means that explaining EIB data can be encapsulated within the attentional-bottleneck framework.

Acknowledgements This work was supported an Australian Research Council Future Fellowship (FT170100021) awarded to S.C.G. and Discovery Project (DP190103103) to M.E.

Open practices statement Raw data are publicly available here: https:// osf.io/dhuqw/.

\section{References}

Barnes, L. L. B., Harp, D., \& Jung, W. S. (2002). Reliability generalization of scores on the Spielberger State-Trait Anxiety Inventory. Educational and Psychological Measurement, 62(4), 603-618. https://doi.org/10.1177/0013164402062004005

Carrasco, M. (2011). Visual attention: the past 25 years. Vision Res, 51(13), 1484-1525. https://doi.org/10.1016/j.visres.2011.04.012

Chun, M. M., \& Potter, M. C. (1995). A two-stage model for multiple target detection in rapid serial visual presentation. $J$ Exp Psychol Hum Percept Perform, 21(1), 109-127.

Ciesielski, B. G., Armstrong, T., Zald, D. H., \& Olatunji, B. O. (2010). Emotion modulation of visual attention: categorical and temporal characteristics. PLoS One, 5(11), e13860. https://doi.org/10.1371/ journal.pone. 0013860

Desimone, R., \& Duncan, J. (1995). Neural mechanisms of selective visual attention. Annu Rev Neurosci, 18, 193-222. https://doi.org/ 10.1146/annurev.ne.18.030195.001205

Endler, N. S., \& Kocovski, N. L. (2001). State and trait anxiety revisited. $J$ Anxiety Disord, 15(3), 231-245.

Goodhew, S. C., \& Edwards, M. (2019). Translating experimental paradigms into individual-differences research: Contributions, challenges, and practical recommendations. Consciousness and Cognition, 69, 14-25. https://doi.org/10.1016/j.concog.2019.01.008

Kennedy, B. L., \& Most, S. B. (2015). The Rapid Perceptual Impact of Emotional Distractors. PLoS One, 10(6), e0129320. https://doi.org/ 10.1371/journal.pone.0129320

Kennedy, B. L., Pearson, D., Sutton, D. J., Beesley, T., \& Most, S. B. (2018). Spatiotemporal competition and task-relevance shape the spatial distribution of emotional interference during rapid visual processing: Evidence from gaze-contingent eye-tracking. Atten Percept Psychophys, 80(2), 426-438. https://doi.org/10.3758/ s13414-017-1448-9

Keysers, C., \& Perrett, D. I. (2002). Visual masking and RSVP reveal neural competition. Trends Cogn Sci, 6(3), 120-125.

Koster, E. H., Crombez, G., Verschuere, B., Van Damme, S., \& Wiersema, J. R. (2006). Components of attentional bias to threat in high trait anxiety: Facilitated engagement, impaired disengagement, and attentional avoidance. Behav Res Ther, 44(12), 1757-1771. https://doi.org/10.1016/j.brat.2005.12.011

Kurdi, B., Lozano, S., \& Banaji, M. R. (2017). Introducing the Open Affective Standardized Image Set (OASIS). Behav Res Methods, 49(2), 457-470. https://doi.org/10.3758/s13428-016-0715-3

Lang, P. J., Bradley, M. M., \& Cuthbert, B. N. (2005). International affective picture system (IAPS) : affective ratings of pictures and instruction manual. Gainesville, Fla.: NIMH, Center for the Study of Emotion \& Attention.

Mogg, K., Bradley, B. P., Miles, F., \& Dixon, R. (2004). Time course of attentional bias for threat scenes: Testing the vigilance-avoidance hypothesis. Cognition and Emotion, 18(5), 689-700. https://doi. org/10.1080/02699930341000158 
Most, S. B., Chun, M. M., Widders, D. M., \& Zald, D. H. (2005). Attentional rubbernecking: cognitive control and personality in emotion-induced blindness. Psychon Bull Rev, 12(4), 654-661.

Most, S. B., Smith, S. D., Cooter, A. B., Levy, B. N., \& Zald, D. H. (2007). The naked truth: Positive, arousing distractors impair rapid target perception. Cognition and Emotion, 21(5).

Most, S. B., \& Wang, L. (2011). Dissociating spatial attention and awareness in emotion-induced blindness. Psychol Sci, 22(3), 300-305. https://doi.org/10.1177/0956797610397665

Ohman, A., Flykt, A., \& Esteves, F. (2001). Emotion drives attention: detecting the snake in the grass. $J$ Exp Psychol Gen, 130(3), 466478.

Onie, S., \& Most, S. B. (2017). Two roads diverged: Distinct mechanisms of attentional bias differentially predict negative affect and persistent negative thought. Emotion, 17(5), 884-894. https://doi.org/10.1037/ emo0000280
Spielberger, C. D., Gorsuch, R. L., Lushene, R., Vagg, P. R., \& Jacobs, G. A. (1983). Manual for the State-Trait Anxiety Inventory. . Palo Alto, CA: Consulting Psychologists Press.

Wang, L., Kennedy, B. L., \& Most, S. B. (2012). When emotion blinds: a spatiotemporal competition account of emotion-induced blindness. Front Psychol, 3, 438. https://doi.org/10.3389/fpsyg.2012.00438

Wang, L., \& Most, S. B. (2016). The cost of seeing the meaning: Conceptual processing of distractors triggers localized target suppression. Visual Cognition, 24(9-10), 473-486. https://doi.org/10. 1080/13506285.2017.1321076

Wyble, B., \& Swan, G. (2015). Mapping the spatiotemporal dynamics of interference between two visual targets. Atten Percept Psychophys, 77(7), 2331-2343. https://doi.org/10.3758/s13414-015-0938-x

Publisher's note Springer Nature remains neutral with regard to jurisdictional claims in published maps and institutional affiliations. 